\title{
Plasmon resonance and electric field amplification of crossed gold nanorods
}

\author{
M.B. Cortie ${ }^{*}$, N. Stokes and A. McDonagh \\ Institute for Nanoscale Technology, University of Technology Sydney, \\ PO Box 123, Broadway NSW 2007, Australia
}

\begin{abstract}
Here we explore the unusual plasmon resonances of crossed gold nanorod structures of varying geometries. Using numerical simulations, we show that the resonances of simple rods are hybridized and blue-shifted in the composite structures and that these structures are surrounded by spatially extended and high intensity electric fields. This attribute suggests several potential uses for these shapes, for example as a nano-antenna for the generation of two-photon fluorescence.
\end{abstract}

PACS: 41.20.Jb, 42.79.Ci, 73.20.Mf, 78.20.Bh

Keywords: gold nanorods, plasmon resonance, electric field, spectrally-selective structure

\footnotetext{
* Corresponding author.

E-mail address: michael.cortie@uts.edu.au
} 


\section{References}

[1] M. J. Tierney, C. R. Martin, J. Phys. Chem. 93(8) (1989) 2878.

[2] C. A. Foss, G. L. Hornyak, J. A. Stockert, C. R. Martin, J. Phys. Chem. 96(19) (1992) 7497.

[3] B. Nikoobakht, M. A. El-Sayed, Chem. Mater. 15 (2003) 1957.

[4] K. Ueno, V. Mizeikis, S. Juodkazis, K. Sasaki, H. Misawa, Opt. Lett. 30(16) (2005) 2158.

[5] Y.-Y. Yu, S.-S. Chang, C.-L. Lee, C. R. C. Wang, J. Phys. Chem. B 101 (1997) 6661.

[6] D. Pissuwan, S. M. Valenzuela, M. B. Cortie, Biotech. Genetic Engng. Rev. 25 (2008) 93.

[7] X. Huang, I. H. El-Sayed, W. Qian, M. A. El-Sayed, J. Am. Chem. Soc. 128(6) (2006) 2115.

[8] X. Xu, T. Gibbons, M. B. Cortie, Gold Bull. 39(4) (2006) 156.

[9] J. W. M. Chon, C. Bullen, P. Zijlstra, M. Gu, Adv. Func. Mater. 17(6) (2007) 875.

[10] X. Zhang, H. Liu, J. Tian, Y. Song, L. Wang, Nano Lett. 8(9) (2008) 2653.

[11] M. Sukharev, T. Seideman, Nano Lett. 6(4) (2006) 715.

[12] J. Pérez-Juste, B. Rodriguez-Gonzalez, P. Mulvaney, L. M. Liz-Marzan, Adv. Func. Mater. 15 (2005) 1065.

[13] M. Cortie, X. Xu, M. Ford, Phys. Chem. Chem. Phys. 8 (2006) 3520.

[14] S. W. Prescott, P. Mulvaney, J. Appl. Phys. 99 (2006) 123504.

[15] M. B. Cortie, X. Xu, M. J. Ford, Phys. Chem. Chem. Phys. 8 (2006) 3520.

[16] B. N. Khlebtsov, N. G. Khlebtsov, J. Phys. Chem. C 111 (2007) 11516.

[17] X. Xu, M. B. Cortie, Adv. Func. Mater. 16(16) (2006) 2170.

[18] E. K. Payne, K. L. Shuford, S. Park, G. C. Schatz, C. A. Mirkin, J. Phys. Chem. B 110 (2006)

2150 .

[19] P. K. Jain, K. S. Lee, I. H. El-Sayed, M. A. El-Sayed, J. Phys. Chem. B. 110 (2006) 7238.

[20] M. B. Cortie, X. Xu 2006, 2006 International Conference on Nanoscience and Nanotechnology Proceedings, Brisbane, Australia, C. Jagadish, G. Q. M. Lu (eds.), p. 81.

[21] P. K. Jain, S. Eustis, M. A. El-Sayed, J. Phys. Chem. B. 110(37) (2006) 18243.

[22] H. Wang, T. B. Huff, D. A. Zweifel, W. He, P. S. Low, A. Wei, J.-X. Cheng, Proc. Natl. Acad. Sci. U.S.A. 102(44) (2005) 15752.

[23] R. A. Farrer, F. L. Butterfield, V. W. Chen, J. T. Fourkas, Nano Lett. 5(6) (2005) 1139.

[24] I. Cohanoschi, F. E. Hernández, J. Phys. Chem. B 109 (2005) 14506.

[25] W. Wenseleers, F. Stellacci, T. Meyer-Friedrichsen, T. Mangel, C. A. Bauer, S. J. K. Pond, S. R. Marder, J. W. Perry, J. Phys. Chem. B 106 (27) (2002) 6853.

[26] N. Stokes, A. M. McDonagh, M. B. Cortie, Gold Bull. 40(4) (2007) 310.

[27] D. Johannsmann, D. Lemke, Infrared Phys. 26 (1986) 215.

[28] B. T. Draine, P. J. Flatau, J. Opt. Soc. Am. A 11(4) (1994) 1491.

[29] B. T. Draine, P. J. Flatau, User Guide for the Discrete Dipole Approximation Code DDSCAT 7.0, http://arxiv.org/abs/0809.0337., September 2008.

[30] A. Brioude, X. C. Jiang, M. P. Pileni, J. Phys. Chem. B 109 (2005) 13138.

[31] K. L. Kelly, E. Coronado, L. L. Zhao, G. C. Schatz, J. Phys. Chem. B 107 (2003) 668.

[32] V. Myroshnychenko, J. Rodríguez-Fernández, I. Pastoriza-Santos, A. M. Funston, C. Novo, P. Mulvaney, L. M. Liz-Marzán, F. J. G. d. Abajo, Chem. Soc. Rev. 37 (2008) 1792

[33] B. M. Nebeker, J. L. d. 1. Peña, D. Hirleman, J. Quant. Spectrosc. Radiat. Transfer 70 (2001)

[34] M. A. Yurkin, A. G. Hoekstra, J. Quant. Spectrosc. Radiat. Transfer 106 (2007) 558.

[35] B. T. Draine, P. J. Flatau, J. Optical Soc. of America A X (2009) in press.

[36] J. Liu, B. Cankurtaran, L. Wieczorek, M. J. Ford, M. B. Cortie, Adv. Func. Mater. 16(11) (2006) 1457.

[37] B. M. I. Van der Zande, M. R. Bohmer, L. G. J. Fokkink, C. Schonenberger, J. Phys. Chem. B 101 (1997) 852.

[38] S.-S. Chang, C.-W. Shih, C.-D. Chen, Langmuir 15 (1999) 701.

[39] B. M. I. van der Zande, M. R. Böhmer, L. G. J. Fokkink, C. Schönenberger, Langmuir 16 (2000)

451.

[40] F. Kim, J. H. Song, P. Yang, J. Am. Chem. Soc. 124(48) (2002) 14316.

[41] C. J. Murphy, T. K. Sau, A. M. Gole, C. J. Orendorff, J. Gao, L. Gou, S. E. Hunyadi, T. Li, J. Phys. Chem. B 109 (2005) 13857. 
[42] J. Perez-Juste, I. Pastoriza-Santos, L. M. Liz-Marzan, P. Mulvaney, Coordin. Chem. Rev. 249 (2005) 1870.

[43] O. G. Tovmachenko, C. Graf, D. J. v. d. Heuvel, A. v. Blaaderen, H. C. Gerritsen, Adv. Mater. 18 (2006) 91. 


\section{Introduction}

There is currently a keen interest in the optical properties of gold and silver ellipsoids, nanocylinders and nanorods, and their potential use in optical nano-devices of various kinds. This is primarily due to the optical anisotropy of these nanoparticles. Early work examined the properties of nano-cylinders of gold produced by deposition within porous alumina templates [1,2], but in the last decade wet chemical methods for the synthesis of high quality gold nanorods have also become available [3]. Nanorods can also be produced by lithographic techniques [4], although these tend towards a parallelepiped shape. Gold nanorods with diameters in the range of 10 to $30 \mathrm{~nm}$ and aspect ratios (length of rod divided by its diameter) of up to 10 manifest two plasmon resonances with light. These are the transverse and longitudinal resonances generated by oscillations of the electric field that are respectively orthogonal or parallel to the long axis of the nanoparticle. The wavelength at which the longitudinal resonance occurs is tunable by control of the aspect ratio and can be varied from the mid-visible wavelengths to as long as $1600 \mathrm{~nm}$ in the near infrared [5] by increasing the aspect ratio. This has encouraged investigations into the application of nanorods in medicine and biology [6, 7], spectrally selective coatings [8], and optical data storage [9]. Another interesting property of nanorods, one that is absent from nanospheres or nanoshells, is a strong dependence of the optical properties on the polarization of the incident light [4]. Not only can aligned arrays of nanorods act as polarizing filters [10], but the reverse also holds and a particular plasmon resonance in a structure containing a nanorod can be switched on or off by control of the polarization of the incident light [11], or by a control of the orientation of the rods themselves. Significant dichroism and/or colour change can be achieved by this means $[12,13]$. Resonances on single rods have been investigated as a function of the size, orientation, shape, and composition of the rods [14-17]. Multipole modes are observable in rods of sufficient diameter and aspect ratio, although these were not studied much until very recently $[16,18]$. However, a diameter of at least $40 \mathrm{~nm}$ and aspect ratio of more than $\sim 4$ is necessary before such modes are resolvable [16]. The interactions between closely spaced nanorods have also been investigated [15, 19-21]. In general, end-to-end interactions cause a red-shift in the longitudinal plasmon resonance, while a side-by-side configuration is associated with a modest blue shift. Two- 
photon phenomena in noble metal nanoparticles have also recently become of interest, both in respect of luminescence of the particle itself [22, 23] or of a dye located near the particle [24, 25].

While much interesting further work remains to be done with individual rods and their optical properties, here we examine a rather different platform for plasmonic phenomena and devices: conjoined nanorod pairs that intersect each other at varying angles (see Fig. 1). These structures possess a lower degree of symmetry compared to ordinary nanorods and are therefore expected to have more complex plasmon resonances. More importantly, however, they can be accurately produced and positioned in large-scale arrays by electron beam lithography [26]. This is not possible with nanoparticles produced by wet chemistry, which are furthermore never monodisperse in size. The precision and flexibility in design associated with lithographically-produced arrays of plasmonicallyactive shapes invites their investigation for a variety of potential new applications. We show here that such structures can be used to produce additional and interesting plasmonic resonances that are not possible in systems of discrete nanorods, and that the electric fields associated with such resonances extend relatively far out into space.

One application that we envisage for arrays of conjoined nanorods is as a nanoantenna to amplify two-photon absorption in a suitable fluorescent dye. Arrays of the crossed rod shapes will also be suitable for use as optical filters with spectral-, orientation- and polarisation-dependent functionality, as optical sensors operating in refractometric mode, or as the components of near-field plasmonic circuitry. Actually, the infrared-screening properties of micron-scale gold meshes, a micron or two wide and crossed at $90^{\circ}$, have been known for decades [27]. However, there has been very little prior investigation of their nanoscale analogues, possibly because lithographic preparation of subwavelength structures has only become available comparatively recently and the optical properties of arrays of such objects has been hitherto largely unknown. Here we present numerical simulations of the optical and electro-magnetic properties of a series of crossed rod structures. A concurrent experimental investigation of the preparation of such structures by electron beam lithography will be reported elsewhere. 


\section{Methodology}

We used the DDSCAT software of Draine and Flatau [28, 29] (DDSCAT: Discrete Dipole Approximation for Scattering and Absorption of Light by Irregular Particles) to perform numerical simulations of a series of conjoined gold nanorod structures. The discrete dipole approximation (DDA) approximates the target by an array of polarisable points over which the electromagnetic scattering of an incident periodic wave is essentially solved for each of these points [29]. The volume of solid materials in the targets processed by this software is expressed in the form of an effective radius, $\mathrm{a}_{\mathrm{eff}}$, defined as the radius of the sphere with the same volume as that of all the solid material in the target. DDSCAT provides accurate simulations of electromagnetic scattering from targets provided that $2 \pi a_{\mathrm{eff}} / \lambda<25$. However, convergence is slow unless $(|m-1|<2)$, where $m$ is the complex refractive index. Metallic targets, in which $|m|>3$ require long computation times. Nevertheless, agreement between calculation and experiment is good, provided the dipole spacing is very small relative to the wavelength, e.g[30-34]. Periodic arrays of targets can also be simulated with DDSCAT [35] although the code does not currently include doing this for arbitrary-shaped targets as a standard. The necessary capability was added for the purpose of the present work by one of the present authors (M.C.).

The shapes examined consisted of crossed pairs of rods that were fused in their central regions with an angular rotation $2 \alpha$ away from parallel alignment. The length of each of the crossed-rod shapes was $80 \mathrm{~nm}$, and the constituent rods had a diameter of $20 \mathrm{~nm}$. A render matrix large enough to give a dipole spacing of about $1 \mathrm{~nm}$ was used. The family of shapes is shown in Fig. 1. The visible and nearinfrared spectra of crossed-rod structures were calculated for the full range of $\alpha\left(0^{\circ}\right.$ through $\left.45^{\circ}\right)$. The nature of the plasmon resonances that occurred was further investigated using animations of the dipoles and calculations of their internal and external electric fields. 


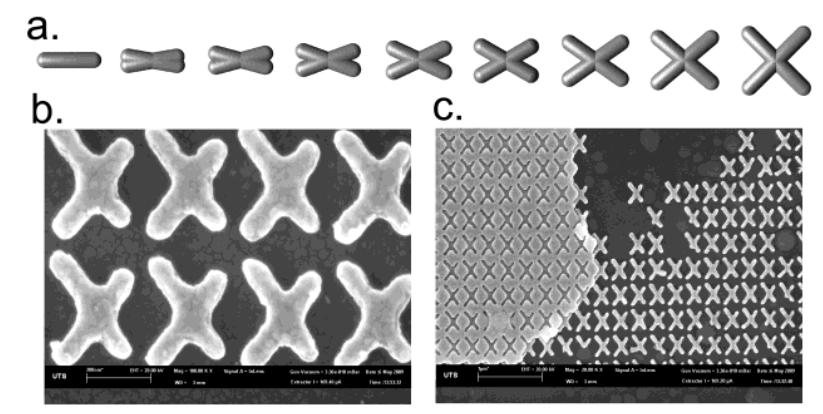

Fig. 1. The conjoined nanorod structures for which the optical properties were calculated. (a) The rotation angles, $\alpha$, of the crossed rods varied from $0^{\circ}$ to $45^{\circ}$ in $5^{\circ}$ intervals. (b) Illustrative example of two-dimensional arrays of gold crosses produced by a combination of electron beam lithography and physical vapour deposition.

(c) Image showing how gold-coated resist is peeled from substrate to leave an array of crosses (Further details of the experimental technique used to produce these coatings will be published elsewhere.)

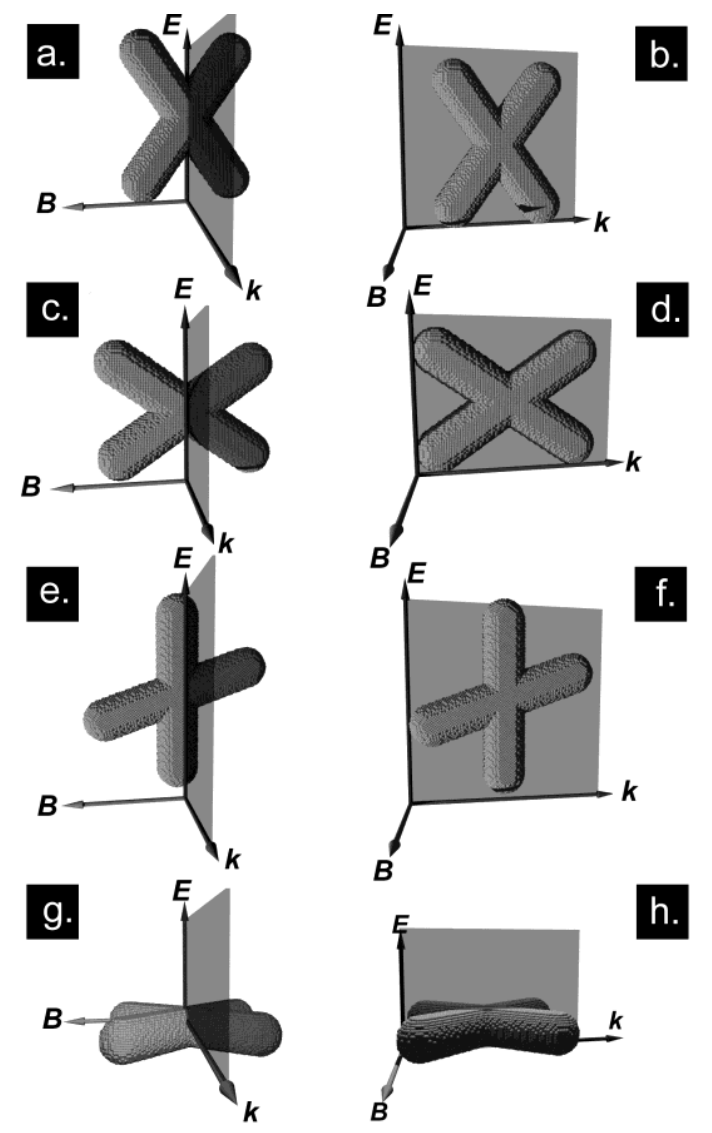

Fig. 2. The different orientations of the electric and magnetic field that are possible for a crossed-rods shape. (a) longitudinal resonance with $\boldsymbol{B}$ in the plane of the target (b) longitudinal resonance with $\boldsymbol{B}$ out-of-plane of the target, (c) transverse resonance with $\boldsymbol{B}$ in-plane (d) transverse resonance with $\boldsymbol{B}$ out-of-plane, (e) electric field oriented along one arm with $\boldsymbol{B}$ in-plane (f) electric field oriented along one arm with $\boldsymbol{B}$ out-of-plane (g) short transverse resonance with $\boldsymbol{B}$ in longitudinal direction of target (h) short transverse resonance with $\boldsymbol{B}$ in transverse direction. 
Fig. 2 shows several different orientations of the targets that are possible with respect to the $\boldsymbol{k}$, $\boldsymbol{E}$ and $\boldsymbol{B}$ vectors of the light. A longitudinal resonance (Fig. 2(a) and 2(b)) would be most prominent when the long axis of the structure is perpendicular to the $\boldsymbol{k}$ vector and parallel to $\boldsymbol{E}$ while a transverse resonance (Fig. 2(c) and 2(d)) would be most prominent when the long axis of the structure is parallel to the $\boldsymbol{k}$ vector of the light. There is also the possibility of aligning one of the arms of the target with the electric field (Fig. 2(e) and 2(f)) and, finally, polarizing the electric field perpendicularly to the plane of the target (Fig. 2(g) and 2(h)). Note that there are two options for $\boldsymbol{k}$ and $\boldsymbol{B}$ for any given orientation of $\boldsymbol{E}$. In general, however, the extinction efficiency of a nanoscale target depends almost entirely on the orientation of $\boldsymbol{E}$, with the direction of $\boldsymbol{k}$ and $\boldsymbol{B}$ being comparatively unimportant [36].

It is worth briefly considering the manner in which the DDA method deals with multipole resonances, and the implications of this. While convergence at wavelengths at which dipole resonances occur is readily established, Fig. 3(a) and 3(b), convergence was slow at the wavelengths at which two or more resonances were simultaneously active, unless an exceedingly fine dipole spacing (for example $<0.5 \mathrm{~nm}$ ) was used. The presence of a spurious bifurcated extinction peak for complex targets in which two resonances were simultaneously active, and their fusion into a single peak when smaller dipole spacings were used, Fig. 3(c), was one of the symptoms of a lack of convergence. The reason for this phenomenon is that individual dipoles in the target can only take on a dipole-type harmonic oscillation (by definition) in the numerical method used. Therefore, the only way in which the numerical method used can simulate a multipole resonance is by mixing domains of different dipolar resonances together so that the ensemble generates the appropriate response. In the present targets these domain mixtures take the form of alternating layers of dipoles, each corresponding to one of the components of the multipole resonance, and with differing phase and amplitude with respect to the planar wavefront of the exciting radiation. Such dipole domains, Fig. 3(d), are absent from simulations of simple dipole resonances, Fig. 3(b). It is the collective interaction of these domains that simulates the multipole electric field oscillation external to the target. Therefore, stable convergence of the numerical solution for multipole resonances requires smaller dipole spacings than would be the 
case for simulations of simple targets, such as nanorods. Nevertheless, the electric field calculated external to the metal nanostructure is expected to be correct.

All figures, except for Fig. 6(c), have a Cartesian coordinate system in which the $x$ direction is horizontal, the $y$ direction is aligned bottom-to-top of page, and $z$ is out-of-plane.
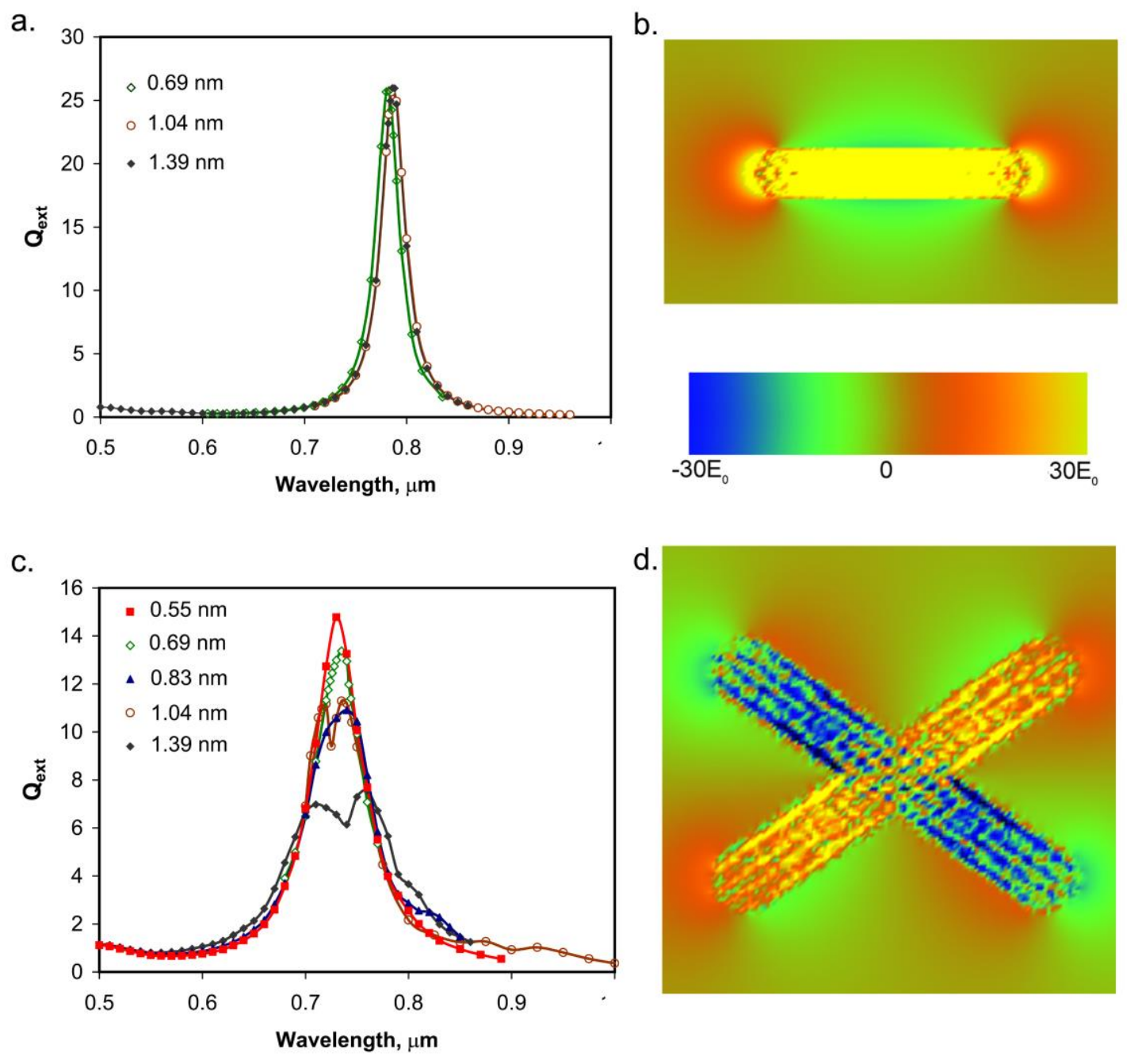

Fig. 3. Effect of dipole spacing on the convergence of the DDA calculations, (a) dipole resonance in a rod of 98 $\mathrm{nm}$ length and $20 \mathrm{~nm}$ diameter inclined at $35^{\circ}$ to $\boldsymbol{k}$, simulated using dipole spacings of $0.69,1.03$, and $1.40 \mathrm{~nm}$, showing that calculations performed with a dipole spacing of $<1.4 \mathrm{~nm}$ produce an extinction peak at $785 \pm 3$ $\mathrm{nm}$, (b) $x$-component of the electric field at $\theta=90^{\circ}$ and $\lambda=782.5 \mathrm{~nm}$ for the rod of (a) revealing the absence of dipole domains, (c) multipole resonance for a crossed rod structure with a half angle of $35^{\circ}$ showing that convergence now requires a dipole spacing $<0.58 \mathrm{~nm}$, (d) $x$-component of the electric field at $\theta=90^{\circ}$ and $\lambda=735$ $\mathrm{nm}$ for the crossed rod target with $\alpha=35^{\circ}$ showing how the internal field of the target with the multipole resonance contains alternating domains of dipoles. The same dipole spacing $(0.69 \mathrm{~nm})$ and colour scheme for the field intensities was used for both (b) and (d)). 


\section{Results and Discussion}

Fig. 4(a) and (b) shows the optical extinction properties calculated at orientations 2(b) and 2(d) for selected instances of the crossed-rods shown in Fig. 1(a). The first peak in Fig. 4(a) is generated by the simplest of the targets: an ordinary gold nanorod with an aspect ratio of 4 and a length of $80 \mathrm{~nm}$, however the extinction of this target is negligible in orientation 2(d) and the first peak shown in Fig. 4(b) is that corresponding to a crossed rod target with $\alpha=15^{\circ}$. The positions and origins of the transverse and longitudinal peaks on the sample with $\alpha=0^{\circ}$ (the simple rod) are consistent with previously reported results [37-42], with a transverse resonance at $\sim 520 \mathrm{~nm}$ being visible in when $\boldsymbol{E}$ is directed across the long axis of the rod, and a longitudinal resonance at $700 \mathrm{~nm}$ becomes visible when $\boldsymbol{E}$ is directed parallel to the long axis.

As $\alpha$ is increased from 0 to $45^{\circ}$ a new resonance appears at about $650 \mathrm{~nm}$ in targets that are in orientation 2(d) (Fig. 4(b)). This new resonance increases in efficiency as the arms of the target are rotated into the longitudinal orientation. On the other hand, as the angle between the rods is increased for orientation 2(b) (Fig. 4(a)), the longitudinal plasmon resonance red-shifts and decreases in efficiency. The decrease is caused by the fact that the arms are increasingly rotated out of the longitudinal orientation. Both resonances red shift as $\alpha$ is increased towards $45^{\circ}$, and converge to become identical at $\alpha=45^{\circ}$. A part of the red shift is clearly produced by the fact that the aspect ratio of the rods increased as $\alpha$ is increased, for purely geometric reasons (the length of the target in the $x$ direction is set at $80 \mathrm{~nm}$, so when $\alpha=45^{\circ}$ the rod will be $80 \sqrt{2} \mathrm{~nm}$, ie. $113.1 \mathrm{~nm}$ long). This can be seen from the position of the peak wavelengths for individual rods of the relevant aspect ratio, superimposed on Fig. 4, which also red shift. Importantly, the peak extinction of the crossed rods is always blue-shifted relative to single rods of the same aspect ratio. This last result can be contrasted to the result reported by Jain et al.[21] for adjacent, non-intersecting rods crossed so that $\alpha=45^{\circ}$. In that case the longitudinal resonances of the individual rods did not interact. The situation is different in our case because the rods are fused together and the electric charge oscillation can freely propagate between the arms of the target. 

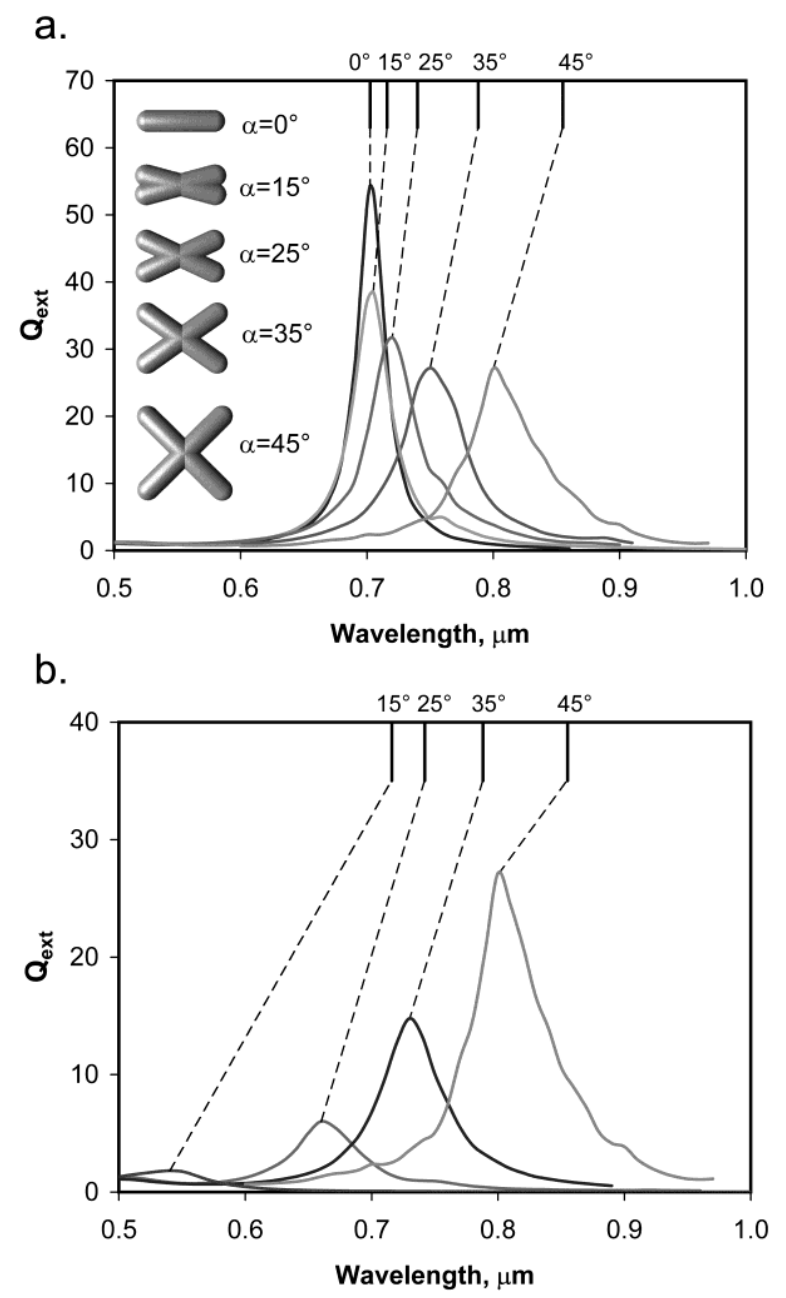

Fig. 4. Effect of $\alpha$ on the calculated extinction efficiencies of X-rod targets in two different orientations. (a) orientation shown in Fig. 2(b) with targets shown as an inset, (b) same targets as in (a) but with orientation shown in Fig. 2(d). Also shown on the graphs are the peak positions of the corresponding isolated, single rods.

Dipole and electric field animations were calculated for the relevant targets in order to elucidate the nature of these resonances. The $45^{\circ}$ resonance will be considered first, as it is in a sense the end member of the series and, furthermore, obviously identical for orientations $2(\mathrm{~b})$ and $2(\mathrm{~d})$. In Fig. 5(a)-(e) we show snapshots of the field taken at a phase angle $(\theta)$ of $90^{\circ}, i e .1 / 4$ of the way through a cycle. It is clear that the overall direction of the dipoles (Fig. 5(a)) is back and forth along the direction of $\boldsymbol{E}$ (aligned here in the $y$ or vertical direction). This produces an approximately dipolar field in each individual arm of the target and the $x$-components of the field must point in opposite directions in each of the arms, Fig. 5(b), since as charge flows upwards it must flow to the left in one arm and to the right in the other. The $y$ components on the other hand all point the same way, Fig. 5(c). There 
was a negligible $\boldsymbol{E}_{\mathrm{z}}$ component, and it is not shown. The overall magnitude of the $\boldsymbol{E}$-field is shown in Fig. 5(d). Overall, the field can be interpreted as shown in Fig. 5(e) for a phase angle of $90^{\circ}$. However, there was also a multipole component which was strongly developed in the vertices of the target. To visualise this in a quantitative sense we placed probe points at the positions shown in Fig. 5(e), and then graphed the overall field strength at the four probe positions as a function of time, Fig. 5(f). It can be seen that the resonances at position 04 lags that at position 01 by $19^{\circ}$ (corresponding to $0.14 \mathrm{fs})$ due to time taken for the planar wavefront to cross the target from left to right. However, the fields at positions 02 and 03 are out of phase by 17 and $34^{\circ}$ respectively with reference to that at position 01. Furthermore, by far their strongest component is aligned along the $y$ direction. Overall, the extinction peak of the complete resonance is considerably blue-shifted compared to individual rods of the same aspect ratio. It is clear that the resonance observed is a new, hybridized multipole oscillation that developed from the superposition of the two individual resonances. 
a.

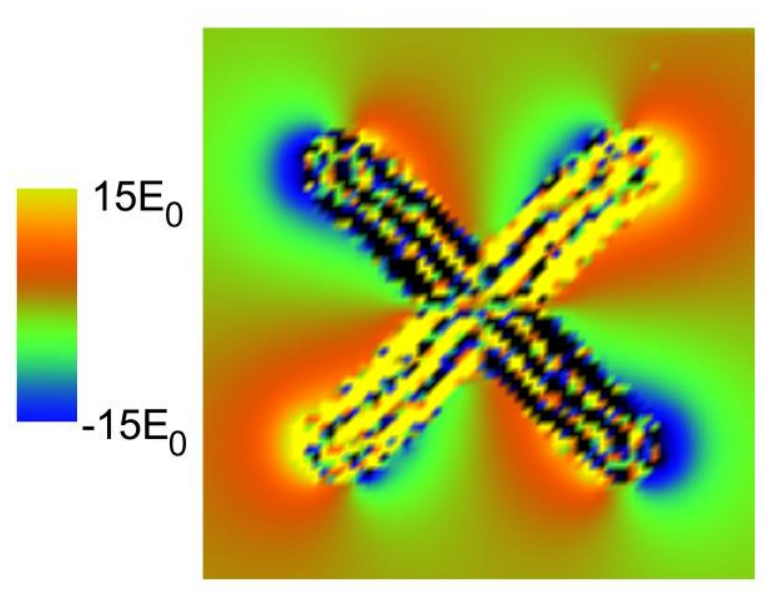

C.

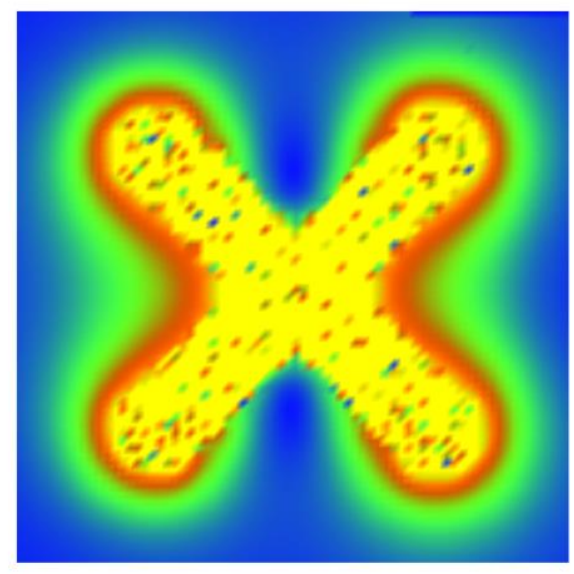

e.

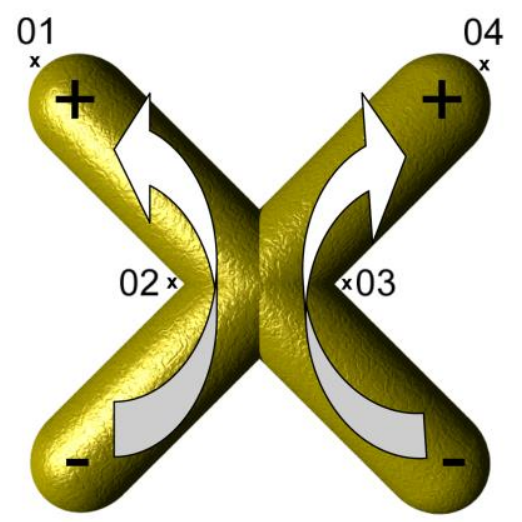

f. b.

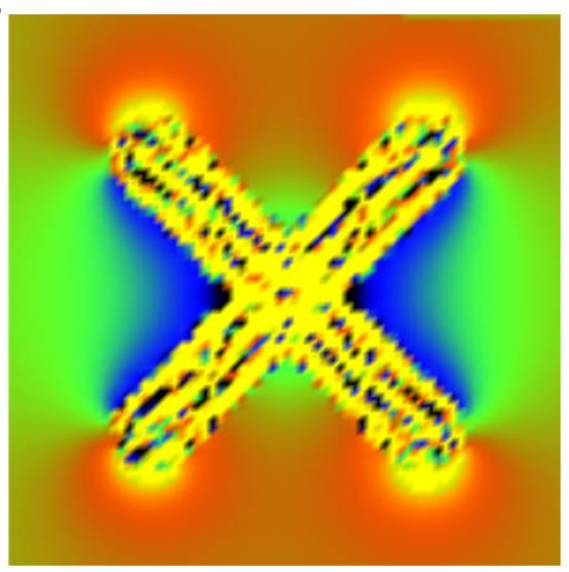

d.
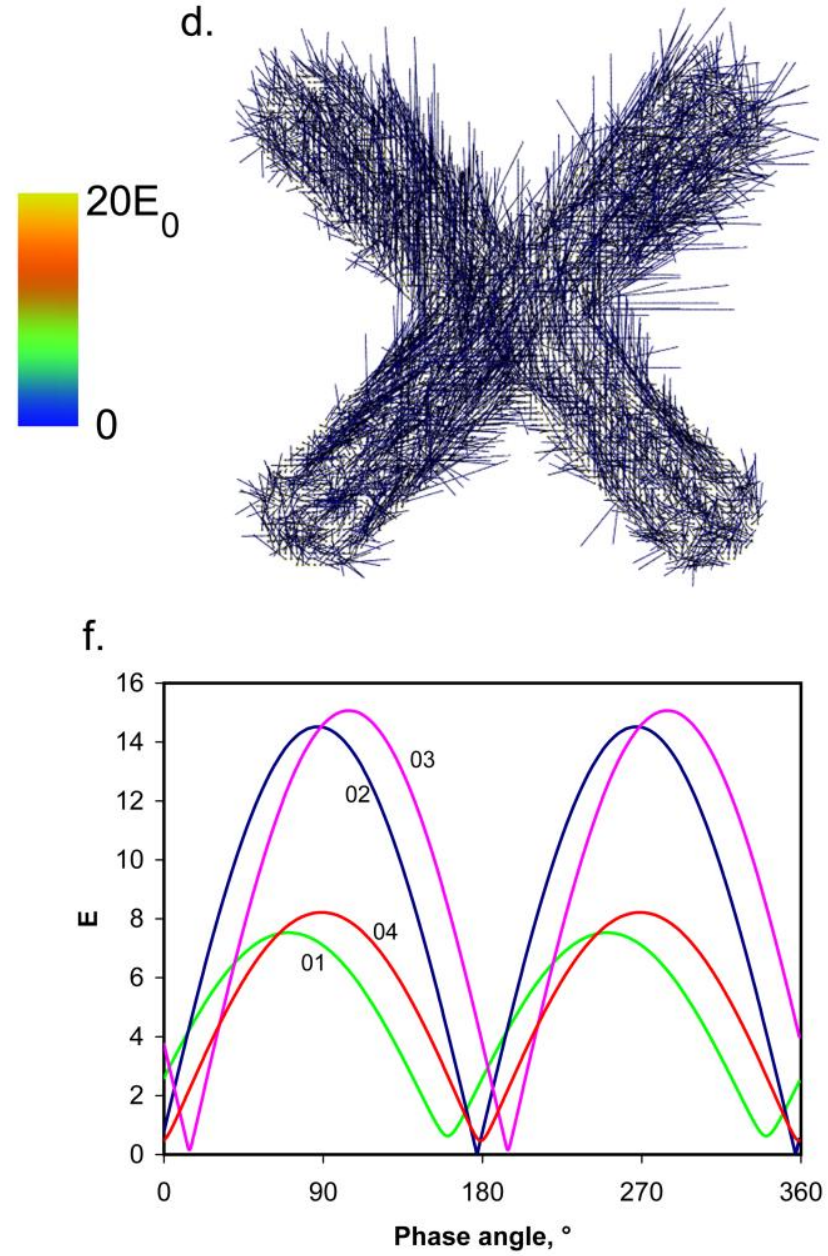

Fig. 5. (a) $\boldsymbol{E}_{\mathrm{x}}$ at phase angle of $90^{\circ}$, on average the field points right in one rod, and left in the other. The 'stripes' within the target are an artefact of the numerical solution, in which layers of dipoles take up one or other of the two resonances being hybridized, (b) $\boldsymbol{E}_{\mathrm{y}}$, (c) $|\boldsymbol{E}|$, (d) dipole vectors at a phase angle of $90^{\circ}$, (e) schematic illustration of dipole resonance, also showing probe positions 01 to 04 , (f) variation of $|\boldsymbol{E}|$ with time at four probe positions. 
Next we consider the nature of the resonances possible in a target with $0<\alpha<45^{\circ}$, using the particular example of the $\alpha=35^{\circ}$ target as a benchmark. Unlike the case for $\alpha=45^{\circ}$, these targets do have a defined longitudinal and transverse orientation and, as shown in Fig. 4, these produce different resonances. The oscillation in these targets could also be considered to be the result of a superposition of a transverse oscillation (in the direction of $\boldsymbol{E}$ ) combined with counter-current longitudinal component running down the length of the two rods. However, the resonances are subtly different for the two orientations. In particular, the resonances in the $\alpha=35^{\circ}$ target develop a stronger longitudinal aspect when in the orientation shown in Fig. 2(b).
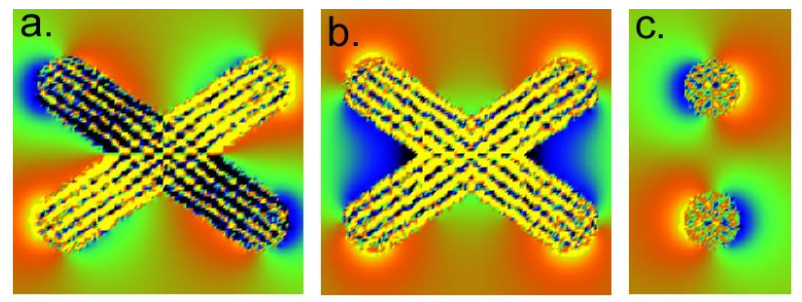

Fig. 6. Resonances in target with half angle of $35^{\circ}$ between arms at a phase angle of $90^{\circ}$ and with the target orientation shown in Fig. 2(d), (a) $\boldsymbol{E}_{\mathrm{x}}$, (b) $\boldsymbol{E}_{\mathrm{y}}$ (c) $\boldsymbol{E}_{\mathrm{z}}$ side view. The intensity scale shown in Fig. 5(a) applies.

It is interesting to consider what happens when $E$ is oriented along one of the arms of a rod in, for example, orientation 2(f), Fig. 7. In this case a strong dipolar longitudinal resonance occurs on one arm of the target, while there is a less intense arm-to-arm resonance elsewhere. This latter resonance peaks at a phase angle that is $20^{\circ}$ later than the first. 
a.

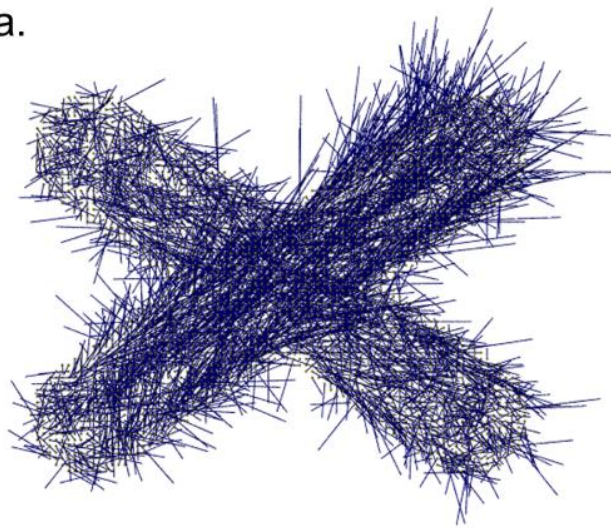

c.

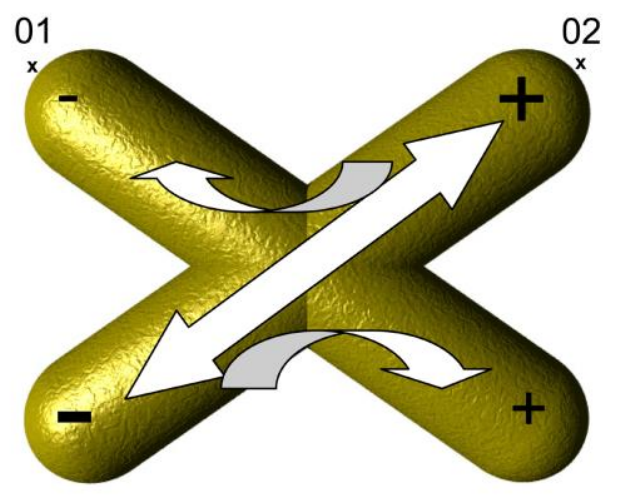

b.

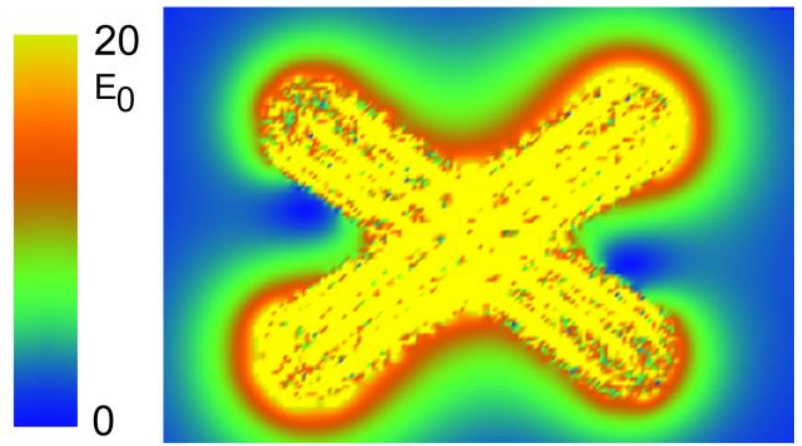

d.

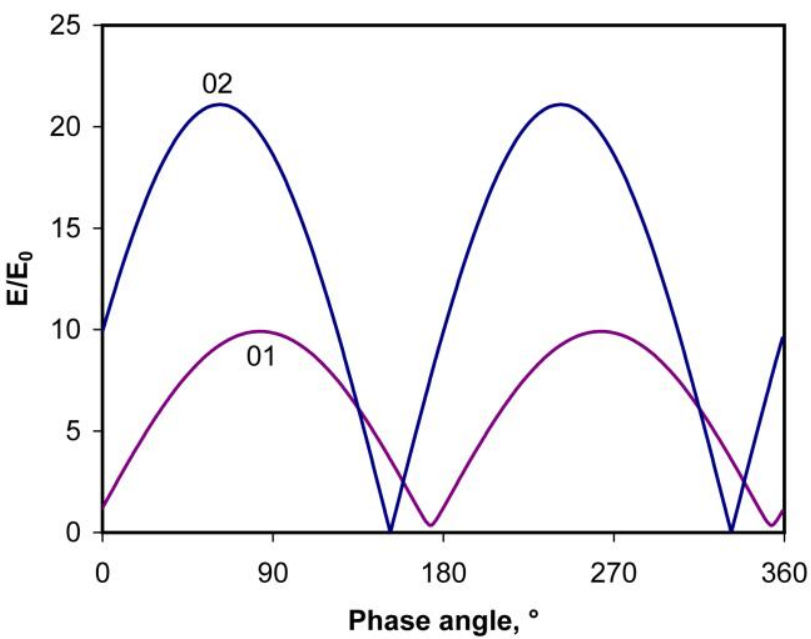

Fig. 7. Effect of placing target so that $\boldsymbol{E}$ points along one of the arms, as shown in Fig. 2(f). (a) dipole depiction of resonance at $\theta=90^{\circ}$ (b) electric field at $\theta=90^{\circ}$, (c) diagram showing the nature of the resonances and probe positions, (d) variation of $|\boldsymbol{E}|$ at the probe positions, showing how the weaker resonance lags the stronger one by $22^{\circ}$.

In these calculations, as the inter-rod angle increases, the amount of gold within the target also increases, due to the decreasing overlap of the crossed rods and to the greater length of the rods as $\alpha$ increases. Therefore the $a_{\text {eff }}$ parameter of the targets also increases as the inter-rod angle is increased. This change has an additional small red-shifting influence of its own, moving the extinction peak a few tens of nanometres as the size is increased for a fixed value of $\alpha$ (Fig. 8). However, it is clear that the primary red-shifting effect in Fig. 4 is due to the increase in inter-rod angle rather than to the small increase in $a_{\text {eff. }}$ 


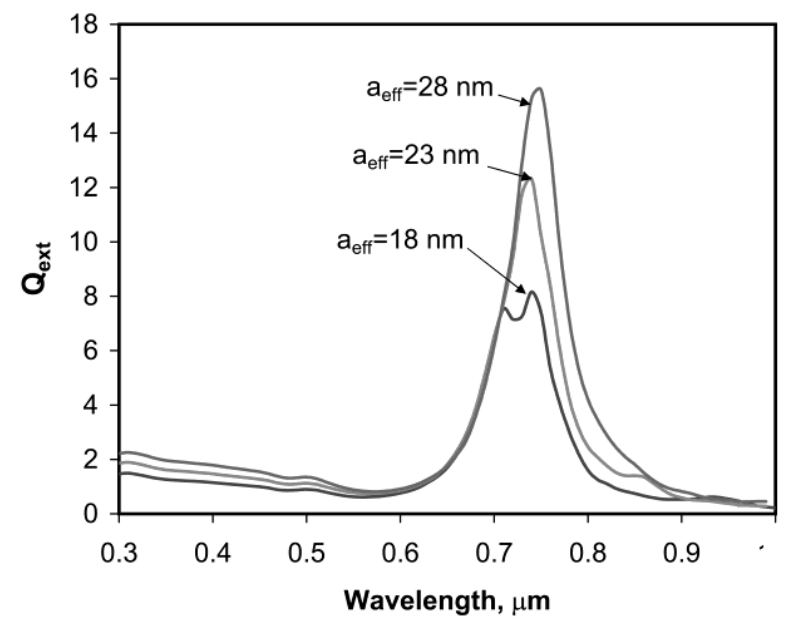

Fig. 8. Effect of target volume on the extinction efficiency of a target with $\alpha=35^{\circ}$ in orientation 2(d). There were insufficient dipoles in the smallest target to ensure the full hybridization of the two resonances.

The preceding discussion has been directed towards the properties of individual targets. Next we examine the effect induced by placing the targets into infinite, periodic arrays. Two orientations of the electric field are important. In the first, the electric field is directed along the length of the array, in the other it is directed across the array. Simulations of the optical properties are shown in Figure 9 for these two polarizations and for various spacings of the targets. In the first case the extinction maxima are red-shifted and in the second polarization the extinction maxima are blue-shifted and broadened. The effects become stronger as the targets are placed closer together. The result in both cases is due to coupling of the plasmons of the individual particles but it is obvious that the coupling takes a different form for the two different polarizations. The results are qualitatively similar to those obtained with simple nanorods. In that case the red-shifting effect was identified as being due to coupling of the plasmons of adjacent particles to form a new lower energy, symmetric plasmon while the blue-shift resulted from coupling to produce a higher energy, anti-symmetric plasmon [21]. We suggest that a similar explanation will apply in the present instance. 


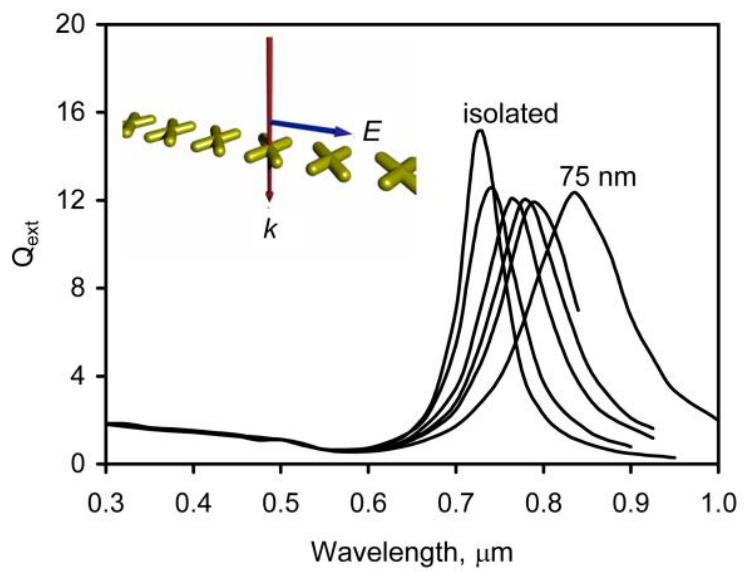

Fig. 9. Simulated optical properties of arrays of crossed rods at various periodic spacings. In this case the rods with a $35^{\circ}$ half-angle have been used to illustrate the point. (a) Electric field directed across the line of targets, periodicity as shown. (b) Electric field directed along the array, the prediction for isolated particles and for a periodic array with a repeat distance of $75 \mathrm{~nm}$ are labelled. Intermediate curves are (from left to right) for repeat units of 150, 100, 90 and $85 \mathrm{~nm}$.

An important question to consider is whether 1-D arrays of crossed rods would possess optical properties that are different to those of coatings of randomly-oriented coating of individual rods, in particular for light incident with the TE polarization (that is, light with $\boldsymbol{E}$ oriented in the plane of the target) onto the coating. The transmission spectra of both types of coating will be characterized by a strong absorption peak, the position of which is tunable, as shown here for the crossed-rods and elsewhere e.g.[8] for the randomly oriented individual rods. However, a coating of rods or crossedrods that are aligned will additionally show a sensitivity to the direction of both the electric and magnetic fields of the light, while a coating made from randomly oriented rods or crossed rods will not. Although spectral selectivity can be obtained by mixing together ordinary nanorods of different aspect ratio, which may have applications in solar screening [8], crossed-rod structures may be attractive in applications that engage with some aspect of the polarization of the incident light or the electric field generated around the targets. For example, a polarizing filter created by a crossed-rod array would have the attributes of being active over only a narrow range of wavelengths, and also have a rotational sensitivity that varies at double the rate of conventional polarizing filters.

The enhanced electric field around such arrays may possibly be even more interesting since it can be exploited, in principle, to amplify either single photon or two-photon fluorescence in suitably 
located dye molecules. The intensity of the electric field as a function of distance from the surface of the targets is shown in Fig.10 for two transects. It is clear that there is a strong and extensive amplification of the electric field between the arms of the target. This is of particular relevance as twophoton phenomena are dependent on $|\boldsymbol{E}|^{2}$ rather than $|\boldsymbol{E}|$. However, excitons that are too close to a metallic nanoparticle are likely to be quenched [43] so the enhanced electric field near the metal surface would only yield useful fluorescence enhancement for dye molecules located at least 10 - 20 nm from the surface [43].

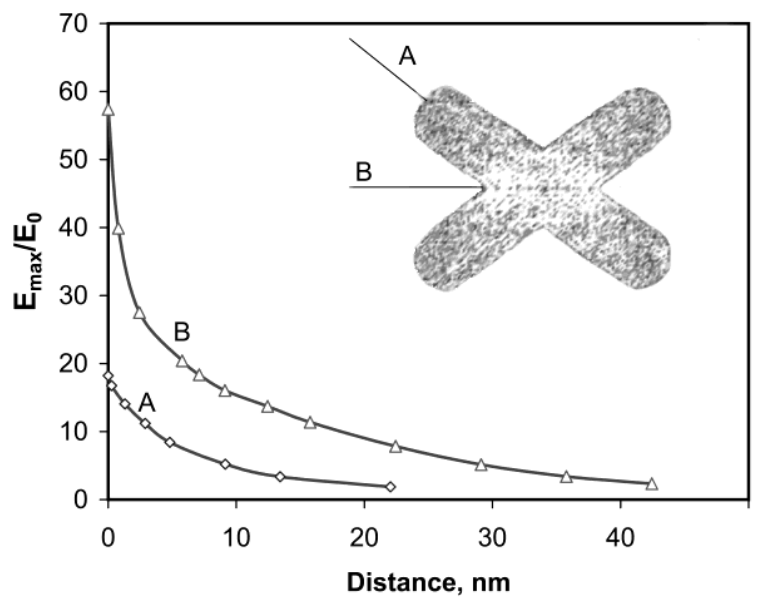

Fig. 10. Variation of electric field intensity as a function of distance from target surface for two transects (A and B) in neighbourhood of crossed rod target with $\alpha=35^{\circ}$ and in orientation 2(d).

\section{Conclusions}

Nanoscale crossed-rod structures made of gold provide a tunable and interesting motif to construct spectrally- and polarization-dependent filters, or with which to amplify two-photon phenomena. A numerical method based on the discrete dipole approximation may be used to calculate the resonance behaviour, however it invokes alternating domains of dipoles within the metal structure in order to reach a solution. These domains reflect the need to hybridize the plasmon resonances in each arm of the structure and reflect the dipole basis of the numerical technique. However, summation of the effect of the individual dipoles produces a reasonable description of the electric field outside the metal structure. The variation of the angle between the rods (and of course the aspect ratio of the arms) provides a useful means of controlling the frequency of the plasmon resonance. Increasing the angle between the arms of the structure causes the peak plasmon resonance to red-shift. The electric field 
distributions in the vicinity of these targets are predicted to be quite complex and have both a high intensity and a spatially extended nature. Although we have presented the results of numerical simulations, the results would be directly transferable to real arrays of gold nanostructures prepared by electron beam lithography. We suggest that such arrays might find practical application as nanoantennas for the generation of two-photon fluorescence in a suitable surrounding medium.

\section{Acknowledgements}

This work was supported by AngloGold Ashanti Australia, the Australian Research Council, Australian Partnership for Advanced Computing (APAC) and the Australian ac3 computer cluster. We thank B.T. Draine and P.J. Flatau for use of their DDA code, DDSCAT, Mr Martin Blaber of UTS for his assistance with computational issues, and Dr Adel Rahmani of UTS for useful discussions. 


\section{Figure Captions}

Fig. 2. The conjoined nanorod structures for which the optical properties were calculated. (a) The rotation angles, $\alpha$, of the crossed rods varied from $0^{\circ}$ to $45^{\circ}$ in $5^{\circ}$ intervals. (b) and (c) Illustrative examples of twodimensional arrays of gold crosses produced by a combination of electron beam lithography and physical vapour deposition. (Further details of the experimental technique used to produce these coatings will be published elsewhere.)

Fig. 2. The different orientations of the electric and magnetic field that are possible for a crossed-rods shape. (a) longitudinal resonance with $\boldsymbol{B}$ in the plane of the target (b) longitudinal resonance with $\boldsymbol{B}$ out-of-plane of the target, (c) transverse resonance with $\boldsymbol{B}$ in-plane (d) transverse resonance with $\boldsymbol{B}$ out-of-plane, (e) electric field oriented along one arm with $\boldsymbol{B}$ in-plane (f) electric field oriented along one arm with $\boldsymbol{B}$ out-of-plane (g) short transverse resonance with $\boldsymbol{B}$ in longitudinal direction of target (h) short transverse resonance with $\boldsymbol{B}$ in transverse direction.

Fig. 3. Effect of dipole spacing on the convergence of the DDA calculations, (a) dipole resonance in a rod of 98 $\mathrm{nm}$ length and $20 \mathrm{~nm}$ diameter inclined at $35^{\circ}$ to $\boldsymbol{k}$, simulated using dipole spacings of $0.69,1.03$, and $1.40 \mathrm{~nm}$, showing that calculations performed with a dipole spacing of $<1.4 \mathrm{~nm}$ produce an extinction peak at $785 \pm 3$ $\mathrm{nm}$, (b) $x$-component of the electric field at $\theta=90^{\circ}$ and $\lambda=782.5 \mathrm{~nm}$ for the rod of (a) revealing the absence of dipole domains, (c) multipole resonance for a crossed rod structure with a half angle of $35^{\circ}$ showing that convergence now requires a dipole spacing $<0.58 \mathrm{~nm}$, (d) $x$-component of the electric field at $\theta=90^{\circ}$ and $\lambda=735$ $\mathrm{nm}$ for the crossed rod target with $\alpha=35^{\circ}$ showing how the internal field of the target with the multipole resonance contains alternating domains of dipoles. The same dipole spacing $(0.69 \mathrm{~nm})$ and colour scheme for the field intensities was used for both (b) and (d)).

Fig. 4. Effect of $\alpha$ on the calculated extinction efficiencies of X-rod targets in two different orientations. (a) orientation shown in Fig. 2(b) with targets shown as an inset, (b) same targets as in (a) but with orientation shown in Fig. 2(d). Also shown on the graphs are the peak positions of the corresponding isolated, single rods.

Fig. 5. (a) $\boldsymbol{E}_{\mathrm{x}}$ at phase angle of $90^{\circ}$, on average the field points right in one rod, and left in the other. The 'stripes' within the target are an artefact of the numerical solution, in which layers of dipoles take up one or other of the two resonances being hybridized, (b) $\boldsymbol{E}_{\mathrm{y}}$, (c) $|\boldsymbol{E}|$, (d) dipole vectors at a phase angle of $90^{\circ}$, (e) schematic 
illustration of dipole resonance, also showing probe positions 01 to 04 , (f) variation of $|\boldsymbol{E}|$ with time at four probe positions.

Fig. 6. Resonances in target with half angle of $35^{\circ}$ between arms at a phase angle of $90^{\circ}$ and with the target orientation shown in Fig. 2(d), (a) $\boldsymbol{E}_{\mathrm{x}}$, (b) $\boldsymbol{E}_{\mathrm{y}}$ (c) $\boldsymbol{E}_{\mathrm{z}}$ side view. The intensity scale shown in Fig. 5(a) applies.

Fig. 7. Effect of placing target so that $\boldsymbol{E}$ points along one of the arms, as shown in Fig. 2(f). (a) dipole depiction of resonance at $\theta=90^{\circ}$ (b) electric field at $\theta=90^{\circ}$, (c) diagram showing the nature of the resonances and probe positions, (d) variation of $|\boldsymbol{E}|$ at the probe positions, showing how the weaker resonance lags the stronger one by $22^{\circ}$.

Fig. 8. Effect of target volume on the extinction efficiency of a target with $\alpha=35^{\circ}$ in orientation 2 (d). There were insufficient dipoles in the smallest target to ensure the full hybridization of the two resonances.

Fig. 9. Simulated optical properties of arrays of crossed rods at various periodic spacings. In this case the rods with a $35^{\circ}$ half-angle have been used to illustrate the point. (a) Electric field directed across the line of targets, periodicity as shown. (b) Electric field directed along the array, the prediction for isolated particles and for a periodic array with a repeat distance of $75 \mathrm{~nm}$ are labelled. Intermediate curves are (from left to right) for repeat units of 150, 100, 90 and $85 \mathrm{~nm}$.

Fig. 10. Variation of electric field intensity as a function of distance from target surface for two transects (A and B) in neighbourhood of crossed rod target with $\alpha=35^{\circ}$ and in orientation 2(d). 